\title{
Neuroimaging in Narcolepsy
}

\author{
A. Bican, I. Bora, O. Algın, B. Hakyemez, V. Özkol and E. Alper
}

Additional information is available at the end of the chapter

http://dx.doi.org/10.5772/25077

\section{Introduction}

Narcolepsy is a chronic neurological disorder, has no specific cause, and is characterized by excessive daytime sleepiness and uncontrollable sleep attacks. In case of strong emotions such as laughter, anger or joy, a cataplexy (sudden loss of muscle tone, lasting for a short period of time) may occur $(1,2)$. Other symptoms of narcolepsy include sleep paralysis and hypnagogic hallucinations $(3,4)$. Narcolepsy can occur in both men and women at any age. The prevalence of narcolepsy shows similar values in North America and Western Europe, varying from $0.02 \%$ to $0.05 \%(5,6)$. HLA DQB1-0602 ratio $85 \%$ and HLA DQA-102 $95 \%$ ratios of people with narcolepsy were found $(7,8)$. In spite of the fact that $99 \%$ of the cases develops sporadically, the risk is 30 to 40 times higher in the first-degree relatives than in the normal population (6).

Hypocretin-1 is reduced in cerebrospinal fluid (CSF) in most people with narcolepsy. Neurons, which contain hypocretins, are widely found in pons, thalamus and cerebral cortex (9). Recent findings indicate a reduction or full-loss of hypocretin cell development in the lateral thalamus of people with narcolepsy (10). Cases are mostly idiopathic (11). Rarely, symptomatic episodes may develop accompanied by brainstem or diencephalon lesions.

There is no gold standard test for the diagnosis of narcolepsy (12). The disorder is often diagnosed on clinical basis (13). A Polysomnogram is appropriate for assessing night-time sleep, while the Multiple Sleep Latency Test (MLST) is employed to evaluate daytime sleep attacks lasting for a short period of time (14).

There is currently a paucity of research evidence relating to magnetic resonance spectroscopy (MRS) and single-photon emission tomography (SPECT) findings in narcolepsy $(9,11,15-17)$. The routine magnetic resonance imaging (MRI) performed on the patients with narcolepsy showed particular differences in various parts of the brain and in pons, while MR indicated variations in T2 weighted images, signal intensity $(17,18)$. Furthermore, according to cerebral perfusion studies, perfusion variations were detected in 
hippocampus, frontal-premotor cortex, pons and thalamus (15). Our purpose in this study is to find out the role of MRI, MRS and SPECT examinations in determining the structural changes in cerebrum and brainstem of people with narcolepsy. In addition, our purpose is also to explore the relationship of clinical and laboratory tests with the results of imaging tests.

\section{Materials and method}

\subsection{Study population}

10 patients with diagnosed narcolepsy (6 males, 4 females) as well as 11 control cases (8 males, 3 females) of similar age group were subjected to MRI, MRS and cerebral SPECT examinations. Average age of people with diagnosed narcolepsy and control group was respectively 37.1 years (range: 23-50 years) and 46 years (range: 34-55 years). Estimated starting age of the patients with disorder was 13.7 years in average (range: 5-25 years). Study protocol was examined and approved by the Ethics Committee of Uludag University. Demographical, clinical and laboratory values of patients with Narcolepsy are given in Table 1.

\begin{tabular}{|l|l|l|l|l|l|l|l|}
\hline Patients & Sexuality & Age & Symptomatology & Cataplexy & $\begin{array}{l}\text { Sleep } \\
\text { Paralysis }\end{array}$ & $\begin{array}{l}\text { Family } \\
\text { history }\end{array}$ & $\begin{array}{l}\text { HLA DQ } \\
\text { B1O602 }\end{array}$ \\
\hline 1 & F & 49 & 25 year & absent & existing & existing & positive \\
\hline 2 & M & 37 & 20 year & existing & existing & absent & positive \\
\hline 3 & M & 32 & 16 year & existing & existing & absent & negative \\
\hline 4 & F & 31 & 10 year & absent & existing & absent & negative \\
\hline 5 & M & 32 & 10 year & absent & existing & absent & negative \\
\hline 6 & M & 42 & 15 year & existing & existing & existing & negative \\
\hline 7 & F & 50 & 10 year & absent & existing & absent & negative \\
\hline 8 & M & 29 & 5 year & existing & absent & existing & negative \\
\hline 9 & M & 23 & 6 year & absent & existing & absent & negative \\
\hline 10 & F & 46 & 20 year & absent & absent & existing & positive \\
\hline
\end{tabular}

Table 1. Demographical, clinical and laboratory values of patients with Narcolepsy.

\subsection{Polysomnogram}

After being assessed in the sleep clinic, the patients were scheduled for nocturnal polysomnograms with subsequent MSLTs. In the meantime, they were advised to avoid having caffeinated foods or drinking alcohol. All nocturnal polysomnograms and next-day sleepiness tests were conducted by means of Grass Telefactor (AS 40 Amplifier system). In the standard polysomnogram, four-channel EEG (C3/A2; C4/A1; O1/A2; O2/A1), twochannel electrooculogram (EOG), submental and anterior tibial muscle electromyogram (EMG), and electrocardiogram (EKG) electrodes were used. Thermistor (oronasal airflow), pulsoxymetry, abdominal and thoracic body sensors were also available. 
The images of the patients were observed through an infrared video camera by a sleep technician, and the records kept. The patients were put to bed at 11:00 pm and waken up at 07:00 am. Sleep stages were scored for each 30-second epoch according to Rechtshaffen and Kales criteria. In MSLT, again four-channel EEG, two-channel EOG, and single-channel EMG records were kept by Grass Telefactor device, accompanied by imaging process by a sleep technician. MSLT was started at 09:00 am in the morning of long nocturnal process, with repetition for 5 times with a 20-minute sleep followed by 20-minute wakefulness. During MSLT, every 30-second sleep epochs were scored considering Sleep-Onset REM, in supervision of a doctor. The patients were diagnosed with narcolepsy according to the International Classification of Sleep Disorders. All of the patients diagnosed with narcolepsy, who had been involved in the study, had short daytime sleep attacks and had not undergone a specific sleep treatment.

\subsection{Cerebral SPECT examinations}

Brain Perfusion SPECT imaging was performed from 02:00 pm to 06:00 pm. All patients were asked to urinate, followed by a 10-minute relaxation period in supine position in an

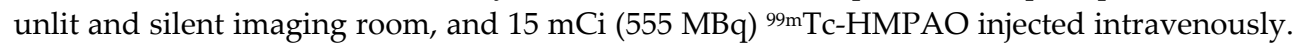
Imaging process was initiated 10 minutes later than injection. In this study, the low-energy, high-resolution, parallel-hole collimator, double detector gamma camera (Millenium VG, GE Medical Systems) was used. Data was gathered with the settings 1.2 zoom, 128x128matrix and centre at $140 \mathrm{keV}, 20 \%$ window interval, at every $5^{\circ}$ rotation angle in step mode for 25 seconds, in total at $360^{\circ}$ rotation in $2 \times 36$ projection; and imaging process lasted 30 minutes. No attenuation or scattering correction is applied. Raw data so gathered was transferred to data-processing and evaluation unit (Entegra Workstation, GE Medical Systems), processed through Butterworth filter (cut-off frequency 0,55 rev./cm; order, 8) and reconstructed in cross-sections with thickness of $3.68 \mathrm{~mm}$, which were parallel to the orbitomeatal line. SPECT cross-sections were evaluated visually at first in orbitomeatal, coronal and sagittal cross-sections. In case of presence of the increased or decreased ${ }^{99 \mathrm{~m} T c-}$ HMPAO retention compared to symmetric areas, the symmetric identical cross-sectional and volumetric areas of interest were determined, semi-quantitative evaluation done, and a standard deviation of 2 or over accepted significant.

\subsection{MR imaging and MR spectroscopy}

All the examinations were performed using 1.5 Tesla MR equipment (Siemens, Magnetom Vision Plus, Erlangen, Germany) with standard head coils, and the study protocol consisted of the following sequences, respectively. Axial-coronal T2-weighted (W) fast spin-echo (FSE) (TR/TE 5400/99), sagittal fluid attenuated inversion recovery (FLAIR) (TR/TE/TI 8400/114/2150), axial-sagittal T1W spin-echo (SE) sequences (TR/TE 550/18), were applied. Field of view (FOV) $24 \mathrm{~cm}, 256 \times 256$ matrix, $5 \mathrm{~mm}$ slice thickness and $1 \mathrm{~mm}$ inter slice gap were obtained. Subsequent to these sequences, a $8 \mathrm{~cm}^{3}$ VOI (volume of interest) was put in frontal cortex, hippocampus, thalamus and pons, and MRS obtained with PRESS sequence 
(TE: 135 ms, TR: $2000 \mathrm{~ms}$, number of acquisitions: 136). Total duration of MR examinations was approximately 20 minutes.

Once imaging process was completed, the routine MR and MRS data available in the MR equipment workstation was analysed by a radiologist (O.A.), who had been unaware of clinical and laboratory findings. The peak values of N-acetyl aspartate (NAA), choline (Cho) and creatine $(\mathrm{Cr})$ as well as their respective ratios were obtained. Total duration of MR examinations was about 10 minutes. All MRI and MRS examinations were evaluated by 2 radiologists (O.A., B.H.), on a randomized basis.

\subsection{Statistical analysis}

The routine MRI, MRS and cerebral SPECT data obtained from the whole cases was compared with clinical and laboratory findings, while the contribution of such examinations to diagnosis evaluated statistically. All statistical analysis was done with statistical programme of SPSS 13.0 software (SPSS Inc., Chicago, IL, USA). The concordance of the data to the normal variation was evaluated with Shapiro-Wilk test. Concordance of normal distribution of all continuous variables was calculated through Shapiro-Wilk test. The relationship between two groups was evaluated with Mann-Whitney U test. The level of statistical significance was set at $\mathrm{P}<0.05$.

\section{Results}

The presence of cataplexy was $40 \%$ (4/10), sleep paralysis $80 \%(8 / 10)$, and family history $40 \%$ (4/10). Two of the patients were brothers. HLA DQ B10602 was positive at a ratio of $30 \%$ (3/10). Average total sleep time of the patients was $397 \mathrm{~min}$. (range: $370-438 \mathrm{~min}$.), average sleep latency $6.5 \mathrm{~min}$. (range: 3-8 min.). Average Epwort sleepiness test was concluded as 10.

The routine cranial MRIs for all cases involved in the study showed no pathological signal variation (Picture 1). In the MRS examinations from frontal cortex and thalamus, no significant statistical difference between NAA/Cr, NAA/Cho, Cho/Cr values of people with narcolepsy and of control group was found ( $>0.05$ ) (Picture 2). Measurements from pons and hippocampus indicated NAA/Cho ratios for narcolepsy group $(1.17 \pm 0.2$ and $0.78 \pm 0.26$, respectively) and for control group $(2.1 \pm 0.76$ and $1.12 \pm 0.15$, respectively) were significantly lower $(\mathrm{p}<0.05)$. Cho/Cr ratios in the level of pons for people with narcolepsy pons $(1.89 \pm 0.52)$ were higher than that for control cases $(1.2 \pm 0.3)$ significantly $(p<0.05)$. In terms of pontine $\mathrm{NAA} / \mathrm{Cr}$ values, no significant difference was detected between narcolepsy group $(2.21 \pm 0.68)$ and control group $(2.46 \pm 0.78) \quad(p>0.05) \quad$ (Picture 3). Measurement from hippocampus showed no significant variation in NAA/Cr and $\mathrm{Cho} / \mathrm{Cr}$ values between narcolepsy group and control group $(\mathrm{p}>0.05)$. MRS values for all cases involved in the study are given in Table 2.

In SPECT examinations, the parietal lobe showed hypoperfusion for one case (Picture 4). SPECT examinations for other cases with diagnosed narcolepsy and for control group were evaluated normal. SPECT data for narcolepsy group is given in Table 3. 


\begin{tabular}{|l|l|l|l|l|}
\hline MRS localization & \multicolumn{1}{|c|}{ Groups } & \multicolumn{1}{c|}{ NAA/Cho } & \multicolumn{1}{|c|}{ NAA/Cr } & \multicolumn{1}{c|}{ Cho/Cr } \\
\hline Pons & Patient with narcolepsy & $1.17 \pm 0.2$ & $2.21 \pm 0.68$ & $1.89 \pm 0.52$ \\
& Controls & $2.1 \pm 0.76$ & $2.46 \pm 0.78$ & $1.2 \pm 0.31$ \\
& Statistical significance & $\mathrm{P}<0.05$ & $\mathrm{P}>0.05$ & $\mathrm{P}<0.05$ \\
\hline Hippocampus & Patient with narcolepsy & $0.78 \pm 0.26$ & $0.95 \pm 0.43$ & $1.19 \pm 0.21$ \\
& Controls & $1.12 \pm 0.15$ & $1.33 \pm 0.21$ & $1.19 \pm 0.15$ \\
& Statistical significance & $\mathrm{P}<0.05$ & $\mathrm{P}>0.05$ & $\mathrm{P}>0.05$ \\
\hline Frontal lobe & Patient with narcolepsy & $2.05 \pm 0.52$ & $2.5 \pm 0.74$ & $1.23 \pm 0.2$ \\
& Controls & $1.76 \pm 0.42$ & $2.26 \pm 1.02$ & $1.09 \pm 0.35$ \\
& Statistical significance & $\mathrm{P}>0.05$ & $\mathrm{P}>0.05$ & $\mathrm{P}>0.05$ \\
\hline Thalamus & Patient with narcolepsy & $1.74 \pm 0.18$ & $1.93 \pm 0.28$ & $1.12 \pm 0.2$ \\
& Controls & $1.95 \pm 0.48$ & $1.9 \pm 0.18$ & $0.97 \pm 0.19$ \\
& Statistical significance & $\mathrm{P}>0.05$ & $\mathrm{P}>0.05$ & $\mathrm{P}>0.05$ \\
\hline
\end{tabular}

Table 2. All cases result of MRS

\begin{tabular}{|l|l|}
\hline Patients & Serebral SPECT (Tc 99m-HMPAO) \\
\hline 1 & Normal \\
\hline 2 & Parietal lobe showed hypoperfusion \\
\hline 3 & Normal \\
\hline 4 & Normal \\
\hline 5 & Normal \\
\hline 6 & Normal \\
\hline 7 & Normal \\
\hline 8 & Normal \\
\hline 9 & Normal \\
\hline 10 & Normal \\
\hline
\end{tabular}

Table 3. SPECT result of patients with Narcolepsy.

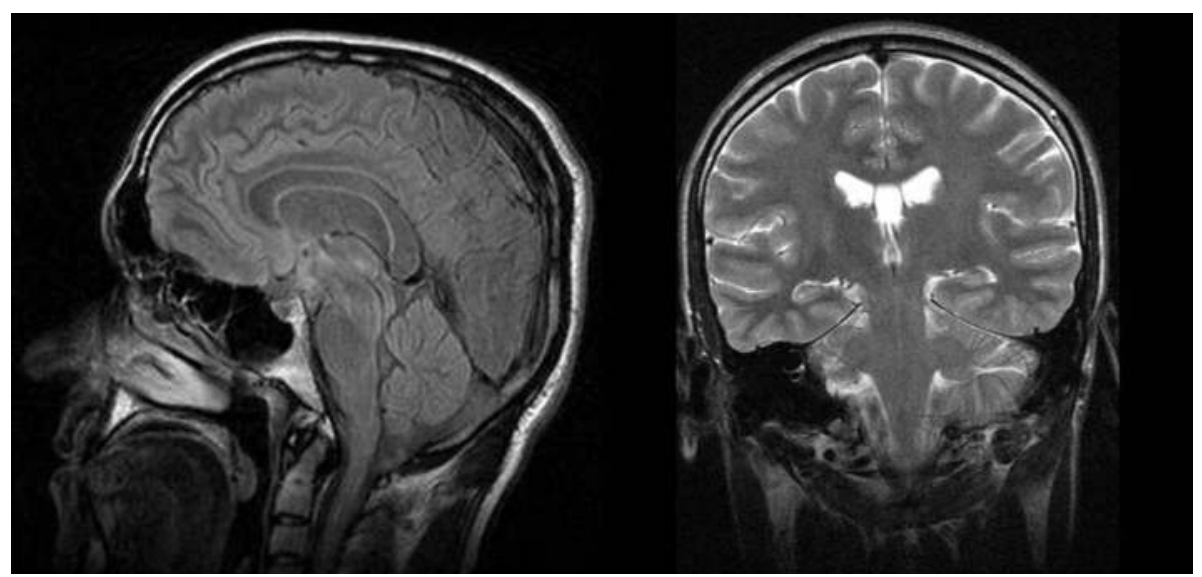

Picture 1. Normal cranial MRI images (sagittal FLAIR and coronal T2W images, respectively) in patient with narcolepsy. 


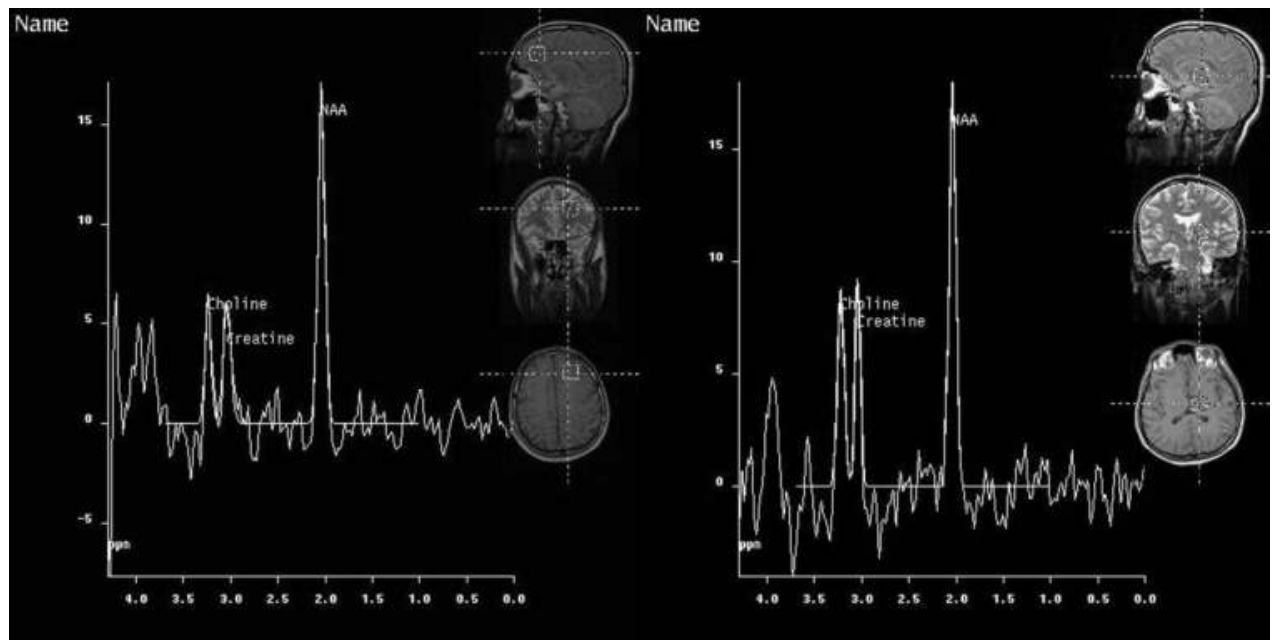

Picture 2. Normal MRS examinations from frontal cortex and thalamus.

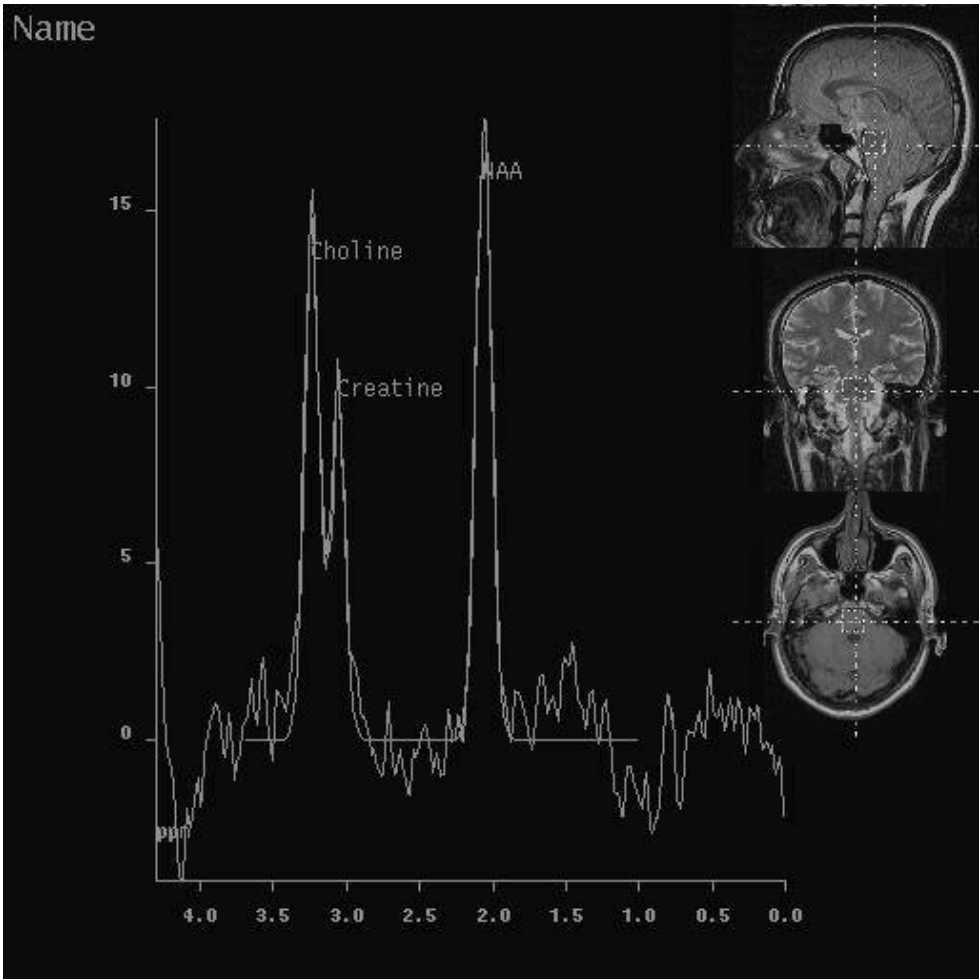

Picture 3. Proton MR spectra in the patient with narcolepsy. N-acetylaspartate (NAA), choline, and creatine peaks are observed. NAA/Cho ratio in this patient was decreased comparing to controls. Also, $\mathrm{Cho} / \mathrm{Cr}$ ratio was increased comparing to controls. 


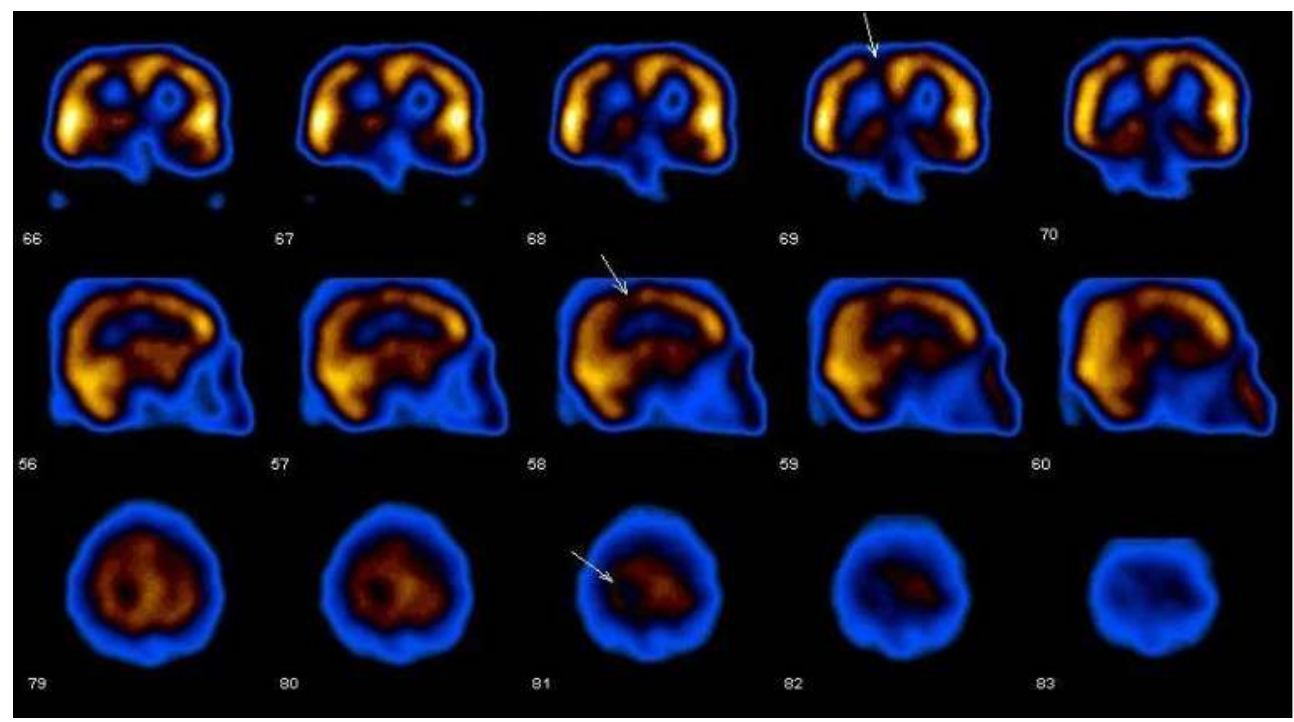

Picture 4. Parietal lobe showed hypoperfusion (2. Patient)

\section{Discussion}

Narcolepsy is mostly an idiopathic disease, and clinical findings are used in diagnosing narcolepsy. There is no gold standard test for the diagnosis of narcolepsy $(9,12)$. Diagnosis criteria include clinical, polysomnogram, multiple sleep latency test (MSLT), and measurement of hypocretin level in cerebrospinal liquid (CSF), which is an invasive method (15). There are a limited number of literatures regarding the use of MRS and SPECT on people with narcolepsy $(11,15,17)$. In most of these literatures the results are controversial $(15,16)$. This may be attributed to the inadequacy of both number and homogeneity of the cases. Our purpose in this study narcolepsy is to find out the role of MRI, MRS and SPECT examinations in determining the structural changes in cerebrum and brainstem in the patients with narcolepsy.

Brain SPECT imaging is a nuclear medicine procedure that allows the clinicians to evaluate the brain function $(15,16)$. Brain perfusion SPECT gives information regarding blood buildup of various anatomic regions in the brain $(12,15,16)$. Reduced activity in hypothalamus, thalamus, prefrontal cortex, parahipocampal gyrus and hippocampus localizations was found during SPECT examinations performed to investigate brain perfusion changes for patients with narcolepsy (15). Specific changes in cerebral blood flow cannot be shown regularly, since the sleep stages in narcolepsy are not fully stabilized (19). In the cataplexic status, high activity was observed in singular gyrus, orbitofrontal and putaminal regions; while, unlike other studies, no activation was shown in pons, amygdala and occipital cortex (20). According to our study, in 9 of 10 cases with diagnosed narcolepsy no perfusion change was detected during SPECT examination. 1 patient showed hypoperfusion in in the right cerebral hemisphere parietal lobe. Based on these findings, it may be suggested that 
SPECT examination has limited contribution to the evaluation of the cases with diagnosed narcolepsy.

One of the most interesting practices of MR procedure is the proton MRS. Based on this technique, information regarding microscopic environments in which protons are available is obtained through chemical shift effect. Protons in the water indicate different release frequencies than those in lactate, adipose tissue or other important biological structures (21, 22). Thus, by the aid of MRS, we are able to determine, on a non-invasive basis, the concentrations of metabolites in brain as well as the metabolite variations occurring for various reasons (21). Today, MRS is used in diagnosing many diseases. MRS plays an important role in the differential diagnosis of infectious, metabolic, ischemic and tumoral diseases (22). Furthermore, by the help of MRS, neuronal damages and metabolite variations, which cannot be shown in brain through the routine MRI, may be also indicated. NAA is a metabolite especially found in neurons and is generally accepted as neuronal marker (23). Reduction in NAA reveals neuronal damage and loss, since the regeneration capacity of neurons is extremely limited $(22,23)$.

In consideration of the fact that narcolepsy may be attributed to neuronal loss or damage in hypothalamus, MRS examinations were performed in relation to hippocampuses for people with narcolepsy. In these studies involving limited number of cases, hypothalamic NAA/Cr was found lower in patients with narcolepsy, compared to control group. Based on this finding, it was emphasized that the central pathology in narcolepsy might be the neuronal loss in hippocampus $(16,17)$. In another study performed through MRS, ventral pontine regions of patients with diagnosed idiopathic narcolepsy were investigated and no variation observed from those of control group $(11,16)$. Certain studies conducted by means of MRI showed variations in several regions of brain and in pons, while those performed through MRI revealed changes in T2-weighted images and in signal intensity (16-18). In the cerebral SPECT study over 25 patients with narcolepsy, Joo, et. al. suggested that hypoperfusion had been found in parahipocampal gyrus, hippocampus, frontal cortex, pons and thalamus. These regions probably reflect cerebral hypocretin system $(15,16)$. For such reasons, MRS measurements from pons, frontal lobe, thalamus and hippocampus were used in our study.

Pontine tegmentum regulates the transition among sleep stages (16). Pons contains reticular formation regulating REM sleep (18). Neuronal damage in this stage is expressed as the main reason of narcolepsy (16). Plazzi, et. al. (18) suggested that pontine T2 hyperintensities detected through MRI are significant in terms of narcolepsy. There are also counterliteratures suggesting that such signal variations are age-dependent non-specific ischemicdegenerative changes $(1,16)$. In the cranial MRI examinations on patients with diagnosed narcolepsy, we detected no significant variation in signal intensity in the pons stage or a pathological finding. Our findings make us think that the routine MRI has no considerable contribution to the diagnosis of narcolepsy. MRI may contribute to the exclusion of other organic lesions, restricting the list of dissepimental diagnoses. In MRS examinations performed with respect to pons, we determined a reduction in NAA/Cho ratios and an increase in $\mathrm{Cho} / \mathrm{Ch}$ rations for patients with narcolepsy, compared to those for control group. These findings reveal the neuronal damage in the pons stage. In a MRS study conducted by Ellis, et. al., no significant variation was found between control group and 
narcolepsy group in terms of metabolite values (11). That study is inconsistent with our findings; however, another MRS study carried out from the pons stage is found to be consonant to our data.

In our study, NAA/Cho ratios in hippocampus were found significantly lower than those of control group. This result makes think that there is a neuronal loss in hippocampuses of patients with narcolepsy for several reasons. Neuronal damage in hippocampus, which is a part of the limbic system, may reveal the emotional instability in patients with narcolepsy. SPECT examinations on people with narcolepsy showed hypoperfusion in the structures forming the limbic system $(15,16)$. Hypoperfusion of the limbic system may become the reason of neuronal damage detected in our study.

The main limitation of our study is the relative adequacy of the number of cases. Furthermore, failure to perform MRS examinations from other regions excluding frontal lobe and from hypothalamus may also feature another limitation of us. We failed to carry out multivoxel MRS due to technical deficiencies in our MR equipment. New wide-series studies in which brain is evaluated globally, also containing multivoxel MRS examinations, and where cases are followed for a long period of time, are required.

As a result, MRI and SPECT examinations have limited contribution to the diagnosis of narcolepsy. MRS appears to be beneficial in diagnosing narcolepsy, indicating those structural changes that cannot be detected through the routine cranial MRI.

\section{Author details}

\section{A. Bican}

Uludag University School of Medicine, Departments of Neurology, Görükle, Bursa, Türkiye Uludag Universitesi Tip Fakultesi, Nöroloji ABD, Görükle, Bursa, Türkiye

İ. Bora

Uludag University School of Medicine, Departments of Neurology, Görükle, Bursa, Türkiye

O. Algin and B. Hakyemez

Uludag University School of Medicine, Departments of Radiology, Görükle, Bursa, Türkiye

V. Özkol and E. Alper

Uludag University School of Medicine, Departments of Nuclear Medicine, Görükle, Bursa, Türkiye

\section{References}

[1] Bassetti C, Aldich MS, Quint DJ. MRI findings in narcolepsy. Sleep 1997; 20:630-631

[2] Aldrich MS. The neurobiology of narcolepsy-cataplexy. Prog Neurobiol 1993;41:533-541

[3] Honda Y. Clinical features of narcolepsy. In: Honda Y, Juji T, ed. HLA in narcolepsy. Berlin: Springer-Verlag, 1988:24-57

[4] Diagnostic Classification Steering Committee. Thorpy MJ CASDA. ICSD-International classification of sleep disorders. Diagnostic coding manual. Rochester, MN: American Sleep Disorders Association; 1990 
[5] National Institutes of Health. National Heart L, and Blood Institute. Narcolepsy. Bethesda: National Institutes of Health, National Heart, Lung and Blood Institute; NIH Publication No. 96-3649,1996

[6] Mignot E. Genetic and familial aspects of narcolepsy. Neurology 1998;50:16-22

[7] Kadatoni H, Faraco J, Mignot E. Genetic studies in the sleep disorder narcolepsy. Genome Res 1998;8:427-434

[8] Mignot E, Hayduk R, Black J, et al. HLA DQB1-602 is associated with cataplexy in 509 narcoleptic patients. Sleep 1997;20:1012-1020

[9] C. Brenneis, E. Brandauer, B. Frauscher, M. Schocke, T.Trieb, W. Poewe, B.Högl Voxelbased morphometry in narcolepsy. Sleep Medicine 2005; 531-536

[10] Nishino S, Ripley B, Overeem S, et al. Hypocretin (orexin) deficiency in human narcolepy. Lancet 2000; 355:39-40

[11] Ellis CM, Simmons A, Lemmens G, Williams SC, Parkes JD. Proton spectroscopy in the narcoleptic syndrome. Is there evidence of a brainstem lesion? Neurology. 1998;50:23-6.

[12] Sturzenegger C, Bassetti CL. The clinical spectrum of narcolepsy with cataplexy: a reappraisal . J. Sleep Res. 2004;13,395-406

[13] Khatami R, Bassetti CL. Narcolepsy. Schweiz Arch Neurol Psychiatr 2003;154:339-348.

[14] Aldrich, M. S, Chervin, R. D and Malow, B. A. Value of the multiple sleep latency test (MSLT) for the diagnosis of narcolepsy. Sleep 1997, 20: 620-629.

[15] Hong SB, Tae WS, Joo EY. Cerebral perfusion changes during cataplexy in narcolepsy patients. Neurology 2006 13;66:1747-9

[16] Dang-Vu TT, Desseilles M, Petit D, Mazza S, Montplaisir J, Maquet P. Neuroimaging in sleep medicine. Sleep Medicine 2007;8:349-372

[17] Lodi R, Tonon C, Vignatelli L, Iotti S, Montagna P, Barbiroli B, Plazzi G.M In vivo evidence of neuronal loss in the hypothalamus of narcoleptic patients. Neurology 2004 26;63:1513-5

[18] Plazzi G, Montagna P, Provini F, Bizzi A, Cohen M, Lugaresi E. Pontine lesions in idiopathic narcolepsy. Neurology 1996;46:1250-4

[19] Asenbaum S, Zeithofer J, Saletu B, Frey R, Brücke T, Podreka I, Deecke L. Technetium99m-HMPAO SPECT imaging of cerebral blood flow during REM sleep in narcoleptics. J Nucl Med. 1995;36:1150-5

[20] Chabas D, Habert MO, Maksud P, Tourbah A, Minz M, Willer JC, Arnulf I. Functional imaging of cataplexy during status cataplecticus. Sleep. 2007;30:153-6

[21] Bonavita S, Di Salle F, Tedeschi G. Proton MRS in neurological disorders. Eur J Radiol 1999; 30:125-131

[22] Yerli H, Ağıldere AM, Özen O, Geyik E, Atalay B, Elhan AH. Evaluation of cerebral glioma grade by using normal side creatine as an internal reference in multi-voxel $1 \mathrm{H}$ MR spectroscopy. Diagn Interv Radiol 2007;13:3-9

[23] Kubas B, Kulak W, Sobaniec W, Walecki J, Lewko J. Proton magnetic rasonance spectroscopy in patients with normal pressure hydrocephalus. The Neuroradiology Journal 2006;19:597-602 